\title{
Influence of fingolimod on basic lymphocyte subsets frequencies in the peripheral blood of multiple sclerosis patients - preliminary study
}

\author{
JULIA RUDNICKA ${ }^{1}$, MICHAE CZERWIEC ${ }^{l}$, EWELINA GRYWALSKA ${ }^{1}$, DOROTA SIWICKA-GIEROBA ${ }^{1,2}$, \\ MONIKA WALANKIEWICZI, AGNIESZKA GRAFKA ${ }^{3}$, MICHAE ZGURSKI ${ }^{l}$, AGATA SURDACKA ${ }^{1,3}$, \\ HALINA BARTOSIK-PSUJEK ${ }^{4}$, JACEK ROLINSSKI
}

${ }^{1}$ Department of Clinical Immunology and Immunotherapy, Medical University of Lublin, Poland

${ }^{2}$ Department of Anesthesiology and Intensive Care, Medical University in Lublin, Poland

${ }^{3}$ St. John's Cancer Center, Lublin, Poland

${ }^{4}$ Department of Neurology, Medical University in Lublin, Poland

\begin{abstract}
Background: Fingolimod is a drug administered orally to adult patients treated for relapsing remitting course of multiple sclerosis (MS). Mode of action of fingolimod is based on intense S1P1 receptor stimulation and "arresting" lymphocytes in lymphatic organs. Objective of the research was to assess changes in the frequencies of basic lymphocyte subsets in patients treated for multiple sclerosis with the use of fingolimod.

Material and methods: Study group comprised of 25 previously untreated adult patients with MS. Venous blood samples were collected from each patient before and one month, three months and six months after treatment initiation. Peripheral blood lymphocyte immunophenotype was assessed with a set of monoclonal antibodies bounded to appropriate fluorochromes and flow cytometer FACSCalibur. Statistical analysis of the results was conducted using Statistica 9.0 software.

Results: Before fingolimod administration median of lymphocyte subsets percentage in each patient was in reference range. After 1 month of treatment we noticed significant changes in frequencies of following lymphocyte subsets: NK cells $-51.22 \%(p=0.016), T C D 4+$ cells $-11.58 \%(p=0.01)$, $T C D 4+: T C D 8+$ cells ratio $-0.61(p=0.005)$. After 3 and 6 months of treatment there was further increase of deviation from normal state.

Conclusions: The use of fingolimod is associated with profound changes in lymphocyte subsets distribution, which might bear a risk of the development of cellular immune deficiency symptoms.
\end{abstract}

Key words: fingolimod, multiple sclerosis, MS, lymphocytes $T, T C D 4+: T C D 8+$ cells ratio.

(Cent Eur J Immunol 2015; 40 (3): 354-359)

\section{Introduction}

Multiple sclerosis (MS) is a chronic central nervous system (CNS) disease. It is characterized by the infiltration of the brain and spinal cord by inflammatory cells, the presence of demyelination areas, axonal degeneration and glial cells destruction [1-3]. Multiple sclerosis is an autoimmune disease resulting from the collapse of the immune system tolerance. Immunity balance loss is based on the presence of the pathological immune response. It leads to cross reactions with the organism's own protein. In the case of MS, these are myelin antigens [4-6]. The incorrect recognition of organism's own antigens or an antigen imitating one's own takes place on the level of the lymph node [7]. From that place autoreactive lymphocytes (espe- cially CD4+ cells) find their way to the circulatory system. Afterwards, they migrate through the blood-brain barrier to infiltrate the brain and spinal cord. The result is the destruction of myelin sheaths of axons by various immune system cells like B cells and cytotoxic macrophages [8].

The fundamental element of pathological changes in MS is the demyelination of neurons. There are four characteristic demyelination models presented in the literature: demyelination by $\mathrm{T}$ cells and macrophages, demyelination by antibodies and complement activation, demyelination through distal oligodendropathy also known as oligodendrocyte apoptosis and demyelination by primary oligodendrocyte degeneration $[9,10]$.

The molecular pathogenesis of MS expertise has enabled identification of cells and their surface molecules

Correspondence: Ewelina Grywalska, MD, PhD, Assoc. Prof., Department of Clinical Immunology and Immunotherapy, Medical University of Lublin, e-mail: dsiw@wp.pl 
making them the target of therapy [11]. Currently there are several kinds of patient treatment: symptomatic treatment, the treatment of the disease relapse (mainly with the usage of corticosteroids) and long-term treatment (with the usage of immunomodulating and immunosuppressive medications). Multiple sclerosis is a chronic disease, therefore long-term therapy constitutes the basis which apart from maintaining the patient's well-being should protect the patient from the disease relapses. Immunomodulating treatment uses interferon $\beta-1-\mathrm{a}$ and $\beta-1-\mathrm{b}$, mitoxantrone, glatiramer acetate, natalizumab and fingolimod [12-14]. The choice of appropriate medication depends on the form and the kind of individual MS symptoms.

Fingolimod is the newest registered drug that significantly differs from the ones used until now. The capsule of fingolimod is administered orally once a day as opposed to preceding drugs administered subcutaneously or intravenously. The mode of action of fingolimod is based on inhibiting autoreactive immunological response on the lymph node level. It bonds compactly with S1P1 receptor which prevents lymphocytes from leaving secondary lymphatic organs. This prevents the penetration to the circulatory system and the invasion of CNS. What is more, the research shows that fingolimod penetrates easily the blood-brain barrier and has neuroprotective effects on the brain tissue [15-17]. Until now there have been many publications on cardiological and pulmonary side effects of fingolimod treatment but there has been no record of its effect on the basic lymphocyte subset frequencies in peripheral blood and the functioning of the immune system.

The objective of the research was to assess changes in the frequencies of basic lymphocyte subsets in patients treated for multiple sclerosis with the use of fingolimod and to determine its clinical implications.

\section{Material and methods}

\section{Patients}

The study group comprised of 25 previously untreated adult patients with MS (10 females, 15 males), hospitalised in the Department of Neurology, Medical University of Lublin, Poland. The patients were diagnosed on the basis of standard criteria. Before treatment initiation, peripheral blood samples from every person were collected and the frequencies of basic lymphocyte subsets were measured. The patients were treated with fingolimod at doses of $0.5 \mathrm{mg} / \mathrm{kg}$ body weight/day during approximately 6 months. The percentages of lymphocyte subsets were assessed one month, three months, and six months after treatment initiation. None of the patients had signs of infection at the time of investigation and for a month before sampling, none had been taking drugs of known influence on the immune system, and none had undergone blood transfusion. Patients with a history of allergic diseases were excluded from the study.
The research protocol was approved by the Ethics Committee of the Medical University of Lublin and all patients gave written informed consent.

Isolation of peripheral blood cells and the detection selected lymphocyte populations.

Venous blood samples were collected from the study patients by venipuncture using sterile, lithium heparin-treated tubes (S-Monovette, SARSTEDT, Aktiengesellschaft \& Co., D-51588 Nubrecht, Germany). Peripheral blood mononuclear cells were aseptically separated by a standard density gradient centrifugation (Gradisol L, Aqua Medica, Poland). The percentage of cells expressing surface markers were analysed. The cells were phenotypically characterised by incubation ( $20 \mathrm{~min}$ in the dark at a room temperature) with a combination of relevant fluorescein isothiocyanate (FITC), phycoerythrin (PE) and CyChrome-labelled monoclonal antibodies (mAbs). Immunofluorescence studies were performed using a combination of the following mAbs: CD3 PE, CD19 PE, CD3 FITC/CD19 PE, CD4 PE, CD8 PE, CD8 FITC/CD4 PE, CD3 FITC/CD16/CD56 PE, CD25 CyChrome and CD69 CyChrome, purchased from BD Biosciences (USA). Three-colour immunofluorescence analyses were performed using a FACS Calibur flow cytometer (Becton Dickinson) equipped with $488 \mathrm{~nm}$ argon laser. A minimum of 10,000 events was acquired and analysed using CellQuest Software. The results were presented as a percentage of CD45+ cells stained with the antibody. The percentage of positive cells was calculated by comparing with the control. Background fluorescence was determined using isotype-matched directly conjugated mouse anti IgG1//gG2 $\alpha$ monoclonal antibodies. The samples were gated on forward scatter vs. side scatter to exclude debris and cell aggregates.

\section{Statistical analysis}

Statistical analysis of the results was conducted using Statistica 9.0. Deviation from normality was evaluated by Kolmogorov-Smirnov test. Data were expressed as the mean value $\pm \mathrm{SD}$, median, minimum and maximum. Differences between groups were assessed using the Mann-Whitney $U$ test. A value $p$ less than 0.05 was considered statistically significant.

\section{Results}

Before fingolimod administration, median of lymphocyte subsets percentage in each patient was as follows: T CD3+ cells - 77.70\%, B CD19+ cells - 8.00\%, NK cells $-11.16 \%$, NKT-like cells $-1.96 \%$, T CD4+ cells $-58.67 \%$, T CD8+ cells $-17.69 \%$, and T CD4+:T $\mathrm{CD} 8+$ cells ratio -3.60 . After 1 month of treatment we noticed significant changes in frequencies of the following lymphocyte subsets: T CD3+ cells $-38.57 \%(p=0.04)$, NK cells $-51.22 \%(p=0.016)$, T CD4+ cells $-11.58 \%$ 
Table 1. Mean percentage lymphocyte subsets in peripheral blood of patients before the initiation of the fingolimod treatment (0) and 1,3, and 6 months after the initiation. Bold marks the most statistically significant changes

\begin{tabular}{lcccc}
\hline & $\mathbf{0}$ & $\mathbf{1}$ month & $\mathbf{3}$ months & $\mathbf{6}$ months \\
\hline CD3+ & 77.69667 & $38.57(p=0.04208)$ & $38.34556(p=0.000051)$ & $41.261(p=0.000377)$ \\
\hline CD19+ & 8.003333 & $3.065(p=0.170483)$ & $3.613333(p=0.007568)$ & $6.69(p=0.766231)$ \\
\hline NK & $\mathbf{1 1 . 1 6 3 3 3}$ & $\mathbf{5 1 . 2 2}(\boldsymbol{p}=\mathbf{0 . 0 1 6 3 6 6})$ & $\mathbf{4 9 . 6 0 2 2 2}(\boldsymbol{p}=\mathbf{0 . 0 0 0 0 0 6})$ & $\mathbf{5 3 . 9 2}(\boldsymbol{p}=\mathbf{0 . 0 0 0 0 1 1})$ \\
\hline NKT-LIKE & 1.963333 & $6.85(p=0.403366)$ & $8.335556(p=0.010023)$ & $5.092(p=0.078942)$ \\
\hline CD4+ & $\mathbf{5 8 . 6 7 3 3 3}$ & $\mathbf{1 1 . 5 7 5}(\boldsymbol{p}=\mathbf{0 . 0 1 2 2 3 1})$ & $\mathbf{1 0 . 5 0 7 7 8}(\boldsymbol{p}=\mathbf{0 . 0 0 0 0 0 0 0 4})$ & $\mathbf{8 . 2 8 4}(\boldsymbol{p}=\mathbf{0 . 0 0 0 0 0 0 0 0 0 8})$ \\
\hline CD8+ & 17.69333 & $19.305(p=0.607614)$ & $25.10222(p=0.061487)$ & $21.405(p=0.148312)$ \\
\hline CD4+:CD8+RATIO & $\mathbf{3 . 6}$ & $\mathbf{0 . 6 0 5}(\boldsymbol{p}=\mathbf{0 . 0 0 5 3 1 4})$ & $\mathbf{0 . 4 6 1 1 1 1}(\boldsymbol{p}=\mathbf{0 . 0 0 0 0 0 0 0 0 0 6})$ & $\mathbf{0 . 3 5 4}(\boldsymbol{p}=\mathbf{0 . 0 0 0 0 0 0 0 0 0 0 0 0 8})$ \\
\hline CD3+/CD25+INCD3+ & 54.79 & $22.695(p=0.036754)$ & $10.42667(p=0.0000001)$ & $9.556(p=0.000000003)$ \\
\hline CD19+/CD25+ & 44.11333 & $36.335(p=0.2347)$ & $21.29222(p=0.000024)$ & $16.733(p=0.00000062)$ \\
INCD19+ & & & & $35.283(p=0.00075)$ \\
\hline CD4+/CD25+INCD4+ & 69.31 & $54.285(p=0.043363)$ & $23.35889(p=0.0000595)$ & $9.075(p=0.070438)$ \\
\hline CD4+/ & 5.11 & $9.065(p=0.56481)$ & $6.795556(p=0.434686)$ & \\
CD25+HIGH(INCD4+) & & & & $37.713(p=0.002907)$ \\
\hline TREG & 12.71333 & $24.55(p=0.454652)$ & $35.32111(p=0.007413)$ & $10.662(p=0.442726)$ \\
\hline CD4+/PD-1+ & 9.116667 & $8.365(p=0.824361)$ & $7.517778(p=0.117409)$ & $7.282(p=0.000429)$ \\
\hline CD8+/PD-1+ & 3.336667 & $10.915(p=0.481269)$ & $8.436667(p=0.125253)$ & \\
\hline CD19+/PD-1+ & & &
\end{tabular}

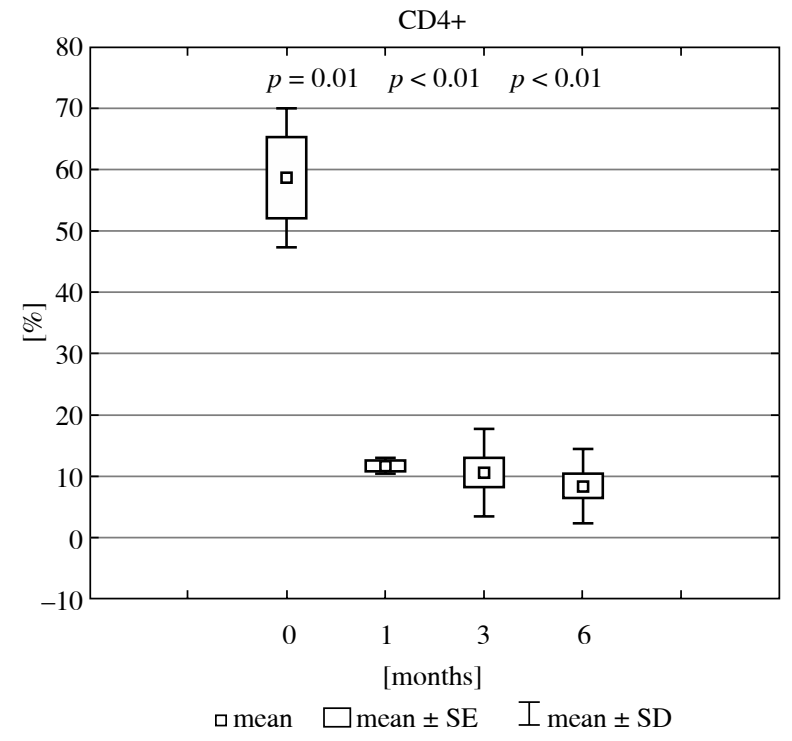

Fig. 1. Mean peripheral blood CD4+ cells percentage in peripheral blood of patients before the initiation of the fingolimod treatment (0) and 1, 3, and 6 months after the initiation

$(p=0.01)$. There was also a decrease in T CD4+:T CD8+ cells ratio $-0.61(p=0.005)$. After 3 months of treatment, there was a further increase in deviation from the normal state, expressed especially in the downgrading of $\mathrm{T}$ CD4+:T CD8+ cells ratio (0.46) compared to the initial state $(p=0.001)$. After 6 months, the median of T CD4+:T $\mathrm{CD} 8+$ cells ratio amounted to 0.35 and the significance compared to the pre-treatment state amounted to $p<0.001$ (Table 1).

The most statistically significant deviations concerned the subsets of CD4+ cells (Fig. 1), NK cells (Fig. 2) and the CD4+:CD8+ cells ratio (Fig. 3).

\section{Discussion}

Although the decrease in the percentage of the CD4+ cells and the decrease in CD4+:CD8+ cells ratio are characteristic of the HIV infection [18], they can also occur during sepsis [19, 20], steroid treatment [21], or Ebola virus infection [22]. Such changes can also result from glutathione and $\mathrm{N}$-acetylcysteine deficiency [23]. They can also be physiological for elderly people [24, 25] and can be idiopathic [26]. The cellular deficiency defect can result in the increased susceptibility to virus, fungus and parasite infections.

Increased susceptibility to the viral infection of serious course was noticed during TRANSFORMS research. The purpose of the research was to assess the effectiveness and safety of fingolimod treatment compared to IFN- $\beta$ treatment in a remitting-relapse type of MS. The research comprised of 1292 patients from 172 clinical centres from 18 countries. In the course of 12 months of drug administration, there were 2 fatal cases. Both concerned patients 


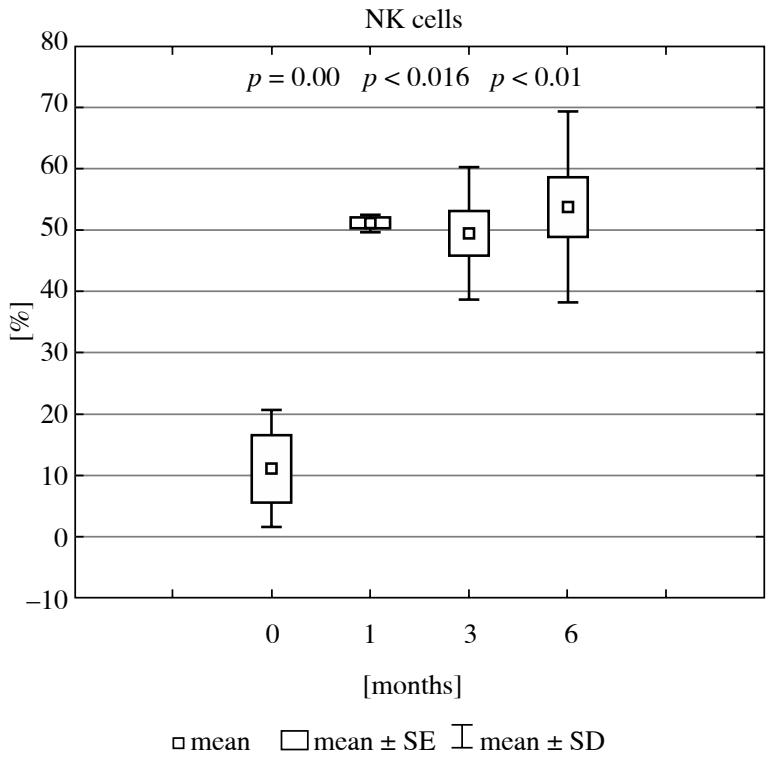

Fig. 2. Mean peripheral blood NK cells percentage in peripheral blood of patients before the initiation of the fingolimod treatment (0) and 1,3, and 6 months after the initiation

treated with fingolimod. One was caused by primary disseminated zoster virus infection in the patient with negative history of varicella infection and negative for viral antibodies. The male patient fell ill after contact with a child with varicella. The fingolimod treatment was continued and intravenous antiviral treatment was administered. Despite antiviral treatment the patient died after 3 days. The second case concerned the decease of a male patient with herpesviral encephalitis. The patient felt ill on 339 day of fingolimod treatment. Because of the suspicion of MS relapse based on symptoms, intravenous methylprednisolone treatment was administered. After a week from the beginning of symptoms of encephalitis, intravenous antiviral treatment was added. The patient died 2 months after starting the treatment [27].

In the following year, a case record of primary disseminated zoster virus infection in a female patient treated with fingolimod for MS was made by Uccelli et al. The patient was administered acyclovir (at first intravenously, then orally). After 15 days cutaneous lesions receded and there were IgM and IgG antibodies against VZV present in blood serum [28].

Another case of viral infection during fingolimod treatment was adduced by Gross et al. A male patient after 42 months of fingolimod treatment had symptoms of left sided facial nerve paralysis and left-side zoster. The patient complained about adverse vertigo which was the result of vestibulocochlear neuritis. He was diagnosed with Ramsay Hunt syndrome type II. Fingolimod treatment was stopped and 21 days of acyclovir treatment was administered. The

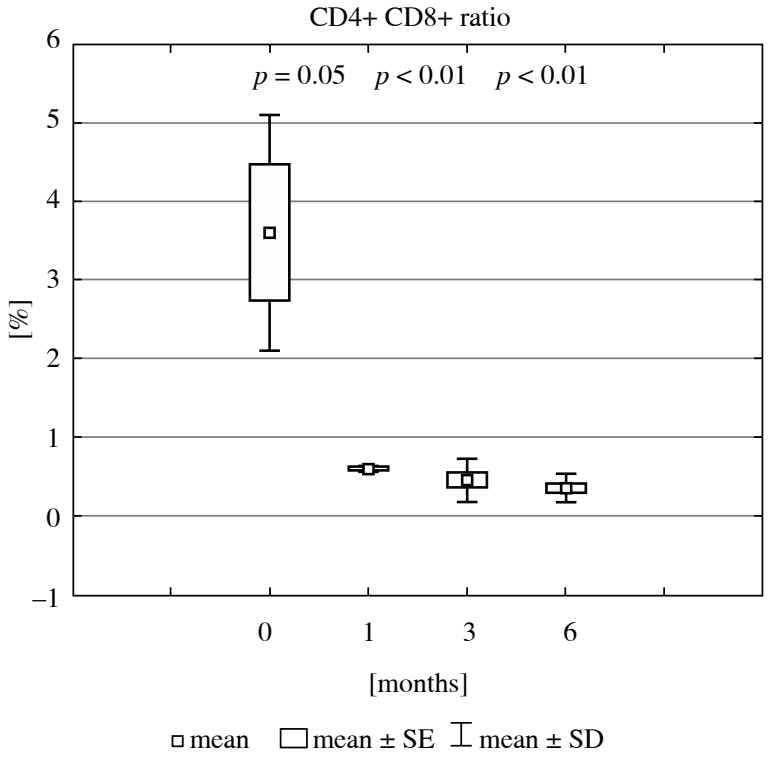

Fig. 3. Mean peripheral blood CD4+ cells:CD8+ cells ratio percentage in peripheral blood of patients before the initiation of the fingolimod treatment ( 0 ) and 1, 3, and 6 months after the initiation

patient recovered. The treatment with $\mathrm{S} 1 \mathrm{P} 1$ inhibitor was discontinued [29].

Ratchford et al. described a case of infection in a fingolimod-treated patient as well. After 3 months of the medicine application blisters with an erythematous ground appeared on a 50-year-old male patient's skin. The patient was diagnosed with zoster and after 4 days of rash appearance, an orally administered acyclovir antiviral treatment was commenced. After another 4 days, the patient suffered generalized seizure resulting in deep coma. The patient needed mechanical breath support with a ventilator. The PCR test for VZV DNA of the cerebrospinal fluid was performed and it confirmed zoster encephalitis. MRI showed 2 spots of ischemic lesions which were recognized as a result of the zoster vasculitis. Intensive pharmacotherapy with levetiracetam, acyclovir (21 days, intravenously) and prednisone (7 days) was commenced. The patient's condition gradually improved and after a month after initial symptoms he regained his state from before the infection [30].

Ricklin et al. took saliva and blood samples from patients to assess antiviral response of patients treated with fingolimod. The samples have been taken from patients before treatment, after 3 months of fingolimod treatment, from patients treated with interferon $\beta$ and patients not treated with drugs. There was also a control group of healthy patients. The results showed increased symptomless reactivation of VZV and EBV in saliva in patients treated with fingolimod in comparison to the control group [31].

In his work, Cervera presented research on the influence of the fingolimod mode of action on frequency of 
infections in patients treated with the drug. He asserted that lymphocytopenia caused by a shift in lymphocyte distribution does not result in the increased incidence of opportunistic infections. Although he noticed such regularity considering the infections caused by the herpes virus family. He took note of the need of the observation of the patients treated with fingolimod considering infections of herpes virus etiology [32].

The changes in basic lymphocyte subset frequencies described in this study concern the decrease in T CD4+ cells percentage and T CD4+:T CD8+ cells ratio. The longer the patients take fingolimod the lower the count of cells involved in cellular response. It can explain the tendency to an increased number of adverse viral infections in fingolimod-treated patients which was described in the literature.

Beside the T cells subsets deviation there is a statistically significant increase in NK cells percentage. Primary immune response is based on the NK cells ability to rapid cytokine and chemokine production and the destruction of a target without earlier hypersensitivity. Until now it has been said that the increase in the NK cell percentage was explained by NK cells leukemia [33], physiological aging process $[34,35]$ and a period of acute virus infection [36].

\section{Conclusions}

The results of our research show a change in basic lymphocyte subsets frequencies which are caused by fingolimod's mode of action. The drug does not allow the lymphocytes to leave the secondary lymph organs through the intense receptor S1P1 inhibition. Not all lymphocyte subsets respond to the drug with the same sensitivity. Presented results suggest that fingolimod affects CD4+ cells the most as the decrease in the number of CD4+ cells was the most significant. It can be concluded from observations that the deviation in lymphocyte subsets frequencies in peripheral blood extends with the length of the treatment with S1P1 receptor inhibitor. These observations correlate with the literature describing clinical side effects of taking fingolimod. Researchers from numerous clinical centers present cases of the patients with adverse virus infections during fingolimod treatment. The infections can be a symptom of deficient cellular immunity resulting from the decrease in CD4+ cells percentage which are a key element of the aforementioned response.

\section{The authors declare no conflict of interest.}

\section{References}

1. Jaśkiewicz E (2004): Epitopes on myelin proteins recognized by autoantibodies present in multiple sclerosis patients. Postepy Hig Med Dosw 58: 472-482.
2. Noseworthy JH, Lucchinetti C, Rodriguez M, Weinshenker BG (2000): Multiple sclerosis. N Engl J Med 343: 938-952.

3. O'Connor KC, Bar-Or A, Hafler DA (2001): The neuroimmunology of multiple sclerosis: possible roles of $\mathrm{T}$ and $\mathrm{B}$ lymphocytes in immunopathogenesis. J Clin Immunol 21: 81-92.

4. Zawada M (2012): Potential pathogens in multiple sclerosis (MS). Postepy Hig Med Dosw 66: 758-770.

5. Dalakas MC (2006): B cells in the pathophysiology of autoimmune neurological disorders: a credible therapeutic target. Pharmacol Ther 112: 57-70.

6. Goverman J (2009): Autoimmune T cell responses in the central nervous system. Nat Rev Immunol 9: 393-407.

7. Lalive PH (2008): Autoantibodies in inflammatory demyelinating diseases of the central nervous system. Swiss Med Wkly 138: 692-707.

8. Lis J, Jarząb A, Witkowska D (2012): Rola mimikry molekularnej w etiologii schorzeń o charakterze autoimmunizacyjnym. Postępy Hig Med Dośw 66: 475-491.

9. Gbadamosi J, Buhmann C, Tessmer W, et al. (2003): Effects of mitoxantrone on multiple sclerosis patients lymphocyte subpopulations and production of immunoglobulin, TNF-alpha and IL-10. Eur Neurol 49: 137-141.

10. Goffette S, van Pesch V, Vanoverschelde JL, et al. (2005): Severe delayed heart failure in three multiple sclerosis patients previously treated with mitoxantrone. J Neurol 252: 1217-1222.

11. Fox EJ (2004): Mechanism of action of mitoxantrone. Neurology 63: 15-18.

12. Szwed M (2014): Mitoxantrone - an anthraquinone antibiotic with antitumor activity applied for the treatment of multiple sclerosis. Postepy Hig Med Dosw 68: 198-208.

13. Bayas A (2013): Improving adherence to injectable disease-modifying drugs in multiple sclerosis. Expert Opin Drug Deliv 10: 285-287.

14. Nogales-Gaete J, Aracena R, Díaz V (2012): Neuropsychological assessment of patients with relapsing remitting multiple sclerosis prior to the use of immunomodulatory drugs. Rev Med Chil 140: 1437-1444.

15. Cruz VT, Fonseca J (2014): Central effects of fingolimod. Rev Neurol 59: 121-128.

16. Groves A, Kihara Y, Chun J (2013): Fingolimod: direct CNS effects of sphingosine 1-phosphate (S1P) receptor modulation and implications in multiple sclerosis therapy. J Neurol Sci 328: 9-18.

17. Chun J, Hartung HP (2010): Mechanism of action of oral fingolimod (FTY720) in multiple sclerosis. Clin Neuropharmacol 33: 91-101.

18. Waters L, Mandalia S, Randell P, et al. (2008): The impact of HIV tropism on decreases in CD4 cell count, clinical progression, and subsequent response to a first antiretroviral therapy regimen. Clin Infect Dis 46: 1617-1623.

19. Piwowar A (2014): The advanced oxidation protein products as potential diagnostic and prognostic factor in diseases of the indicated participation of oxidative stress. Postepy Hig Med Dosw (Online) 68: 446-458.

20. Hotchkiss RS, Tinsley KW, Swanson PE, et al. (2001): Sepsis-induced apoptosis causes progressive profound depletion of B and CD4+ T lymphocytes in humans. J Immunol 166: 6952-6963.

21. Pelekanou A, Tsangaris I, Kotsaki A, et al. (2009): Decrease of CD4-lymphocytes and apoptosis of CD14-monocytes are characteristic alterations in sepsis caused by ventilator-asso- 
ciated pneumonia: results from an observational study. Crit Care 13: R172.

22. Gupta M, Spiropoulou C, Rollin PE (2007): Ebola virus infection of human PBMCs causes massive death of macrophages, CD4 and CD8 T cell sub-populations in vitro. Virology 364: 45-54.

23. Konikowska K, Regulska-Ilow B (2014): The role of diet in multiple sclerosis. Postepy Hig Med Dosw (Online) 68: 325-333.

24. Wikby A, Maxson P, Olsson J, et al. (1998): Changes in CD8 and CD4 lymphocyte subsets, $\mathrm{T}$ cell proliferation responses and non-survival in the very old: the Swedish longitudinal OCTO-immune study. Mech Ageing Dev 102: 187-198.

25. De Paoli P, Battistin S, Santini GF (1988): Age-related changes in human lymphocyte subsets: progressive reduction of the CD4 CD45R (suppressor inducer) population. Clin Immunol Immunopathol 4: 290-296.

26. Walker UA, Warnatz K (2006): Idiopathic CD4 lymphocytopenia. Curr Opin Rheumatol 18: 389-395.

27. Cohen JA, Barkhof F, Comi G, et al. (2010): Oral fingolimod or intramuscular interferon for relapsing multiple sclerosis. N Engl J Med 362: 402-415.

28. Uccelli A, Ginocchio F, Mancardi GL, Bassetti M (2011): Primary varicella zoster infection associated with fingolimod treatment. Neurology 76: 1023-1024.

29. Gross CM, Baumgartner A, Rauer S, Stich O (2012): Multiple sclerosis rebound following herpes zoster infection and suspension of fingolimod. Neurology 79: 2006-2007.

30. Ratchford JN, Costello K, Reich DS, Calabresi PA (2012): Varicella-zoster virus encephalitis and vasculopathy in a patient treated with fingolimod. Neurology 79: 2002-2004.

31. Ricklin ME, Lorscheider J, Waschbisch A, et al. (2013): $\mathrm{T}$-cell response against varicella-zoster virus in fingolimod-treated MS patients. Neurology 81: 174-181.

32. Cervera C (2012): Infections and fingolimod. Rev Neurol 55: 227-237.

33. Soliman DS, Sabbagh AA, Omri HE (2014): Rare aggressive natural killer cell leukemia presented with bone marrow fibrosis - a diagnostic challenge. Springerplus 3: 390.

34. Ligthart GJ, van Vlokhoven PC, Schuit HR, Hijmans W (1986): The expanded null cell compartment in ageing: increase in the number of natural killer cells and changes in T-cell and NK-cell subsets in human blood. Immunology 59: 353-357.

35. Krishnaraj R, Blandford G (1988): Age-associated alterations in human natural killer cells. 2. Increased frequency of selective NK subsets. Cell Immunol 114: 137-148.

36. Adamiak M, Tokarz-Deptuła B, Deptuła W (2014): Characteristic of innate lymphoid cells (ILC). Postepy Hig Med Dosw (Online) 68: 1464-1471. 